\title{
PERINEURAL CAPSAICIN INDUCES THE UPTAKE AND TRANSGANGLIONIC TRANSPORT OF CHOLERATOXIN B SUBUNIT BY NOCICEPTIVE C-FIBER PRIMARY AFFERENT NEURONS
}

\author{
O. OSZLÁCS, G. JANCSÓ, * G. KIS, M. DUX AND \\ P. SÁNTHA \\ Department of Physiology, University of Szeged, Dóm tér 10, \\ H-6720 Szeged, Hungary
}

\begin{abstract}
The distribution of spinal primary afferent terminals labeled transganglionically with the choleratoxin $B$ subunit (CTB) or its conjugates changes profoundly after perineural treatment with capsaicin. Injection of CTB conjugated with horseradish peroxidase (HRP) into an intact nerve labels somatotopically related areas in the ipsilateral dorsal horn with the exceptions of the marginal zone and the substantia gelatinosa, whereas injection of this tracer into a capsaicin-pretreated nerve also results in massive labeling of these most superficial layers of the dorsal horn. The present study was initiated to clarify the role of C-fiber primary afferent neurons in this phenomenon. In L5 dorsal root ganglia, analysis of the size frequency distribution of neurons labeled after injection of CTB-HRP into the ipsilateral sciatic nerve treated previously with capsaicin or resiniferatoxin revealed a significant increase in the proportion of small neurons. In the spinal dorsal horn, capsaicin or resiniferatoxin pretreatment resulted in intense CTB-HRP labeling of the marginal zone and the substantia gelatinosa. Electron microscopic histochemistry disclosed a dramatic, $\sim 10$-fold increase in the proportion of CTB-HRP-labeled unmyelinated dorsal root axons following perineural capsaicin or resiniferatoxin. The present results indicate that CTB-HRP labeling of $\mathrm{C}$-fiber dorsal root ganglion neurons and their central terminals after perineural treatment with vanilloid compounds may be explained by their phenotypic switch rather than a sprouting response of thick myelinated spinal afferents which, in an intact nerve, can be labeled selectively with CTB-HRP. The findings also suggest a role for GM1 ganglioside in the modulation of nociceptor function and pain. (c) 2015 IBRO. Published by Elsevier Ltd. All rights reserved.
\end{abstract}

Key words: spinal cord, C-fiber primary afferents, capsaicin, transient receptor potential vanilloid type 1 receptor, choleratoxin B subunit, plasticity.

*Corresponding author. Tel: + 36-62-545-099; fax: + 36-62-545842. E-mail address: gaborjancso@yahoo.co.uk (G. Jancsó).

Abbreviations: CGRP, calcitonin gene-related peptide; CTB, choleratoxin B subunit; CTB-HRP, HRP conjugated to choleratoxin B subunit; DRG, dorsal root ganglion; HRP, horseradish peroxidase; TMB, tetramethylbenzidine; TMP, thiamine monophosphatase; TRPV1, transient receptor potential vanilloid type 1 receptor; WGA, wheat germ agglutinin; WGA-HRP, HRP conjugated to wheat germ agglutinin.

\section{INTRODUCTION}

Perineural treatment with capsaicin and other vanilloids has been shown to produce selective and long-lasting regional thermal and chemical analgesia and abolition of the neurogenic inflammatory response without affecting non-nociceptive sensory nerves, and autonomic efferent and motor nerves (Jancsó et al., 1980, 1985, 1987a,b; Fitzgerald and Woolf, 1982; Gamse et al., 1982; Jancsó and Such, 1983; Chung et al., 1985; Jancsó and Ambrus, 1994; Kissin, 2008). Capsaicin applied onto peripheral nerves produces this unique effect (which we term nociceptor analgesia) through selective action on nociceptive afferent nerves, although the exact mechanisms involved are not yet fully elucidated (Jancsó et al., 1980, 2011; Gibson et al., 1982; Petsche et al., 1983; Welk et al., 1983; Lynn and Pini, 1984; Jancsó and Lynn, 1987; Holzer, 1991). The defunctionalization of primary afferent neurons which express the transient receptor potential vanilloid type 1 (TRPV1)/capsaicin receptor through the perineural application of capsaicin has become a well-established experimental strategy in pain research to study nociceptive and viscerosensory mechanisms (South and Ritter, 1988; Maggi et al., 1989; Holzer, 1991; Nagy et al., 2004; Jancsó and Sántha, 2015). Vanilloid-induced nociceptor analgesia may be produced in any area of the body by treating the corresponding nerve(s) in a variety of animal species (Gamse et al., 1982), including primates (Chung et al., 1985), and it has therefore been considered as a possible therapeutic approach for the relief of pain of peripheral or neuropathic origin (Jancsó et al., 1980, 2008; Fazen and Ringkamp, 2008; Kissin, 2008; Knotkova et al., 2008). Despite extensive studies on the effects of perineural capsaicin treatment on the morphology, function and neurochemistry of primary afferent neurons, the mechanisms involved in the development of the antinociceptive actions of this treatment are still unclear. Previous studies have revealed a marked decrease in the sensitivity of C-fiber primary afferent neurons toward capsaicin following perineural capsaicin treatment (Jancsó and Lawson, 1990; Jancsó and Ambrus, 1994). More recent studies indicate that this may be explained by massive reductions in the mRNA and protein expressions of the TRPV1 receptor, which confers capsaicin sensitivity on primary sensory neurons (Winter et al., 1988; Caterina et al., 1997; Michael and Priestley, 1999) after perineural treatment with capsaicin (Szigeti et al., 2012). Neuronal tract tracing and histochemical studies involving use of the cholera- 
toxin $B$ subunit (CTB) conjugated with horseradish peroxidase (HRP) revealed that, unlike in control animals, injection of this tracer into a previously capsaicin-treated peripheral nerve resulted in marked transganglionic labeling not only of the deeper layers of the spinal dorsal horn, the termination sites of myelinated primary afferents, but also of the substantia gelatinosa, the main termination site of unmyelinated primary afferents. Since CTB and its conjugates are taken up and transported selectively only by myelinated primary afferent fibers in intact animals (Robertson and Grant, 1985; LaMotte et al., 1991; RiveroMelian and Grant, 1991), the labeling of the substantia gelatinosa after perineural treatment with capsaicin was interpreted in terms of a sprouting response of A-fiber afferents (Mannion et al., 1996). However, subsequent studies have revealed that, following peripheral nerve injury, e.g. nerve transection, not only myelinated A-fibers, but also unmyelinated nociceptive C-fibers take up and transport CTB and its conjugates (Tong et al., 1999; Bao et al., 2002; Jancsó et al., 2002, 2004; Sántha and Jancsó, 2003; Shehab et al., 2003; Jancsó and Sántha, 2004). Electron microscopic studies have furnished direct evidence of the transport of CTB-HRP by unmyelinated dorsal root axons after peripheral nerve transection (Sántha and Jancsó, 2003; Jancsó and Sántha, 2004). It has been concluded, therefore, that the increased labeling by transganglionically transported CTB or its conjugates of the substantia gelatinosa of the spinal dorsal horn after peripheral nerve transection may be explained by a phenotypic switch of nociceptive C-fiber primary sensory neurons rather than a sprouting response of myelinated primary afferents (Tong et al., 1999; Bao et al., 2002; Jancsó et al., 2002, 2004; Hughes et al., 2003; Sántha and Jancsó, 2003; Shehab et al., 2003; Jancsó and Sántha, 2004).

CTB is a highly specific and sensitive biochemical marker of the GM1 ganglioside receptor (Cuatrecasas, 1973; Eidels et al., 1983). Hence, the mechanisms underlying the increased transganglionic labeling of the substantia gelatinosa by CTB-HRP following perineural treatment with capsaicin may be of significance not only in terms of the anatomical changes which might occur after such treatment, but also as regards the possible functional significance of altered levels of neural gangliosides, and in particular GM1. Several lines of evidence indicate involvement of the GM1 ganglioside in nociceptive mechanisms. Besides its suggested role in opiate action (Crain and Shen, 1998), the GM1 ganglioside has recently been shown to be an important regulator of neuronal capsaicin sensitivity and the expression of the TRPV1 receptor (Sántha et al., 2010).

The present study was therefore initiated to clarify the mechanism(s) contributing to the labeling of the substantia gelatinosa by peripherally injected CTB-HRP and to explore the possible implications of GM1 in vanilloid-induced nociceptor analgesia following perineural capsaicin treatment.

\section{EXPERIMENTAL PROCEDURES}

The experiments were approved by the Ethics Committee for Animal Care at the University of Szeged and were carried out in full accordance with the European Communities Council Directive of 24 November 1986 $(86 / 609 / E E C)$. All efforts were made to minimize animal suffering. The number of experimental animals was kept as low as possible.

\section{Perineural treatment with TRPV1 agonists and labeling with CTB-HRP and WGA-HRP}

Adult male Wistar rats weighing $250-270 \mathrm{~g}$ at the start of the experiments were used in this study. The rats were anesthetized with chloral hydrate $(400 \mathrm{mg} / \mathrm{kg}$, i.p., Reanal, Budapest, Hungary). The sciatic nerves were exposed high in the thigh on both sides, and small pieces of gelfoam moistened with $0.1 \mathrm{ml}$ of either a $1 \%$ solution of capsaicin (Fluka, Buchs, Switzerland) or a $0.005 \%$ solution of resiniferatoxin (Sigma-Aldrich $\mathrm{GmbH}$, Steinheim, Germany) or the same volume of vehicle $(6 \%$ ethanol, $8 \%$ Tween 80 in saline) were wrapped around the right and left nerves, respectively. After $20 \mathrm{~min}$, the gelfoam pieces were removed, the wounds were closed, and the rats were returned to the animal house. After 14 days, the animals were anesthetized again, the sciatic nerves were exposed in the midthigh and $1 \mu \mathrm{l}$ of a $1.5 \%$ solution of CTB-HRP or wheat germ agglutinin (WGA)-HRP conjugate (Sigma-Aldrich $\mathrm{GmbH}$, Steinheim, Germany) was injected into each nerve via a Hamilton microsyringe. Three days after the injection of CTB-HRP or WGA-HRP, the animals were deeply anesthetized and perfused transcardially with an aldehyde fixative containing $1.25 \%$ glutaraldehyde and $1 \%$ paraformaldehyde in $0.1 \mathrm{M}$ phosphate buffer ( $\mathrm{pH} 7.4)$, followed by $400 \mathrm{ml}$ of cold phosphate buffer containing $30 \%$ sucrose. The L4-L6 dorsal root ganglia and the L3-L6 segments of the lumbar spinal cord were removed and stored in the sucrose-buffer solution. Serial frozen sections of the dorsal root ganglia (DRGs) $(15 \mu \mathrm{m}$ in thickness) and the spinal cord (60 $\mu \mathrm{m}$ in thickness) were cut on a cryostat and a freezing microtome, respectively. Peroxidase activity was demonstrated by using $3,3^{\prime}, 5,5^{\prime}-$ tetramethylbenzidine (TMB, Sigma-Aldrich $\mathrm{GmbH}$, Steinheim, Germany) as chromogen (Mesulam, 1978).

Size-frequency distribution histograms of CTB-HRPlabeled neurons with clear-cut nuclei were generated by measuring their sizes in representative serial sections of the L5 DRGs of each animal by means of a light microscope equipped with a camera lucida and a digitizing tablet connected to a computerized system. The data are expressed as mean \pm S.D. Statistical analysis of the data was performed with the Student $t$-test. $\quad p<0.05$ was regarded as a statistically significant difference between groups.

\section{Demonstration of thiamine monophosphatase (TMP)}

TMP activity was demonstrated essentially as described earlier (Inomata and Ogawa, 1979), with some modifications to produce a reaction end-product of lead chromate instead of lead sulfide. Briefly, sections of the L4-L5 DRGs and the spinal cord were placed into an incubation solution containing $7.3 \mathrm{mM}$ thiamine monophosphate 
(Sigma-Aldrich $\mathrm{GmbH}$, Steinheim, Germany), $80 \mathrm{mM}$ lead nitrate and $161 \mathrm{mM}$ sucrose in Tris-maleate buffer $(0.01 \mathrm{M}, \mathrm{pH} 5.4)$. After incubation at $37^{\circ} \mathrm{C}$ for 60-90 $\mathrm{min}$, the sections were washed in Tris buffer and treated with $5 \%$ potassium bichromate for $5 \mathrm{~min}$. The sections were then washed again, dehydrated, cleared with xylene and mounted with DePeX. The sections were viewed under a Leica DMLB (Leica Microsystems $\mathrm{GmBH}$, Wetzlar, Germany) light microscope.

Consecutive representative sections of the spinal cord processed for the demonstration of TMP activity and the localization of CTB-HRP were used to assess the effectiveness of capsaicin treatment and for exact location of the somatotopically relating areas of the capsaicin-treated peripheral nerves within the spinal dorsal horn.

\section{Electron microscopic histochemistry}

The types of dorsal root afferents which transport CTBHRP were identified at the ultrastructural level by using electron microscopic histochemistry. In 3 animals, the L5 dorsal roots, in which the majority $(>80 \%)$ of the afferent fibers belongs to the sciatic nerve (Yip et al., 1984), were ligated $12 \mathrm{~h}$ after the injection of CTB-HRP into the intact and capsaicin-treated nerves, respectively. Thirty-six hours later, the animals were perfused and the L5 dorsal roots were removed. Two-to-three millimeter segments were cut from the roots distal to the ligatures and peroxidase activity was demonstrated using TMB as a chromogen and tungstic acid as a stabilizer (Gu et al., 1992). The samples were then reacted with diaminobenzidine and cobaltous chloride, postfixed with osmium tetroxide, dehydrated and embedded in Araldite. Ultrathin sections were cut on a Reichert Ultrotome, contrasted with uranyl acetate and lead citrate and viewed under a Jeol Jem 1010 (Jeol, Tokyo, Japan) electron microscope. Dorsal root axons were counted on screen in randomly selected sections.

\section{RESULTS}

\section{Effects of perineural treatment with capsaicin or resiniferatoxin on the labeling of L5 DRG neurons with CTB-HRP injected into the sciatic nerve}

Injection of CTB-HRP into the sciatic nerve resulted in the labeling of many neurons in the L4-L6 spinal ganglia relating to the control and the capsaicin-treated sciatic nerves. Light microscopic inspection of specimens obtained from L4-L5 ganglia relating to capsaicin- or resiniferatoxin-treated sciatic nerves indicated a marked increase in the number of labeled small neurons as compared with the control (Fig. 1). For quantitative assessment of the proportions and sizes of labeled neurons, quantitative morphometric measurements were performed in representative serial sections of the L5 DRGs, since about $80 \%$ of neurons in those DRGs send their axons into the sciatic nerve (Yip et al., 1984). An analysis of the size-frequency distribution histograms of the L5 DRG neurons revealed a pronounced increase in the number of labeled small neurons after perineural cap- saicin or resiniferatoxin treatment (Fig. 2B-D). In ganglia relating to the control nerve, small and large DRG neurons were labeled; the mean cross-sectional area of labeled neurons amounted to $943.2 \pm 47.88 \mu \mathrm{m}^{2}$. In the L5 spinal ganglia relating to the capsaicin and the resiniferatoxin-treated sciatic nerve, the proportion of small neurons increased significantly, resulting in a significant decrease in the mean neuronal cross-sectional area to $698.2 \pm 27.59 \mu \mathrm{m}^{2}$ and $673.6 \pm 43.44 \mu \mathrm{m}^{2}$, respectively. In a previous study involving quantitative morphometry and a statistical approach, we could separate the large- and small-sized populations of DRG neurons; we demonstrated that, under the experimental conditions also used in the present study, neurons with crosssectional areas smaller or larger than $600 \mu \mathrm{m}^{2}$ can be classified as small and large neurons, respectively (Soares et al., 2002; Szigeti et al., 2012). Statistical evaluation of the data revealed a significant increase in the proportion of small neurons from $44.0 \pm 1.26 \%$ to 66.4 $\pm 0.64 \%$ and $65.6 \pm 1.03 \%$ after perineural treatment with capsaicin and resiniferatoxin, respectively (Fig. 2A). These findings suggested a change in the phenotype of small neurons which acquired the ability to take up and transport CTB-HRP after capsaicin treatment, possibly due to an increase in their GM1 ganglioside level.

\section{Effects of perineural treatment with capsaicin or resiniferatoxin on the labeling of the substantia gelatinosa with CTB-HRP injected into the sciatic nerve}

TMP histochemistry was used to identify the topographical distribution of sciatic primary afferent terminals affected by perineural treatment with the vanilloid compounds. In agreement with previous findings (Ainsworth et al., 1981; Gamse et al., 1982), perineural application of capsaicin and resiniferatoxin resulted in an abolition of TMP activity from the somatotopically appropriate projection territories of the sciatic nerve in the substantia gelatinosa of the spinal dorsal horn (Fig. 3A, B, E, F). In accord with previous findings, injection of CTB-HRP into a control sciatic nerve resulted in intense labeling of the somatotopically corresponding areas of the spinal dorsal horn, with the exceptions of the substantia gelatinosa and the marginal zone in the L3-L6 segments of the spinal cord (Fig. 3C, G). This labeling pattern is characteristic of the distribution of thick myelinated primary afferent fibers in the dorsal horn (Szentágothai, 1964; Réthelyi and Szentágothai, 1973; Brown, 1981; Willis and Coggeshall, 1991; Ramon y Cajal, 1995). In agreement with previous findings (Mannion et al., 1996), following the injection of CTBHRP into a capsaicin-pretreated sciatic nerve, marked labeling was also observed in the somatotopically appropriate areas of the most superficial laminae of the spinal dorsal horn, the substantia gelatinosa and the marginal zone (Fig. 3D, H). In the present study, similar results were obtained following treatment of the sciatic nerve with resiniferatoxin (Fig. 4). These findings were unexpected, since the substantia gelatinosa is the main projection territory of unmyelinated primary afferents, and myelinated primary afferents do not normally terminate in this lamina. 

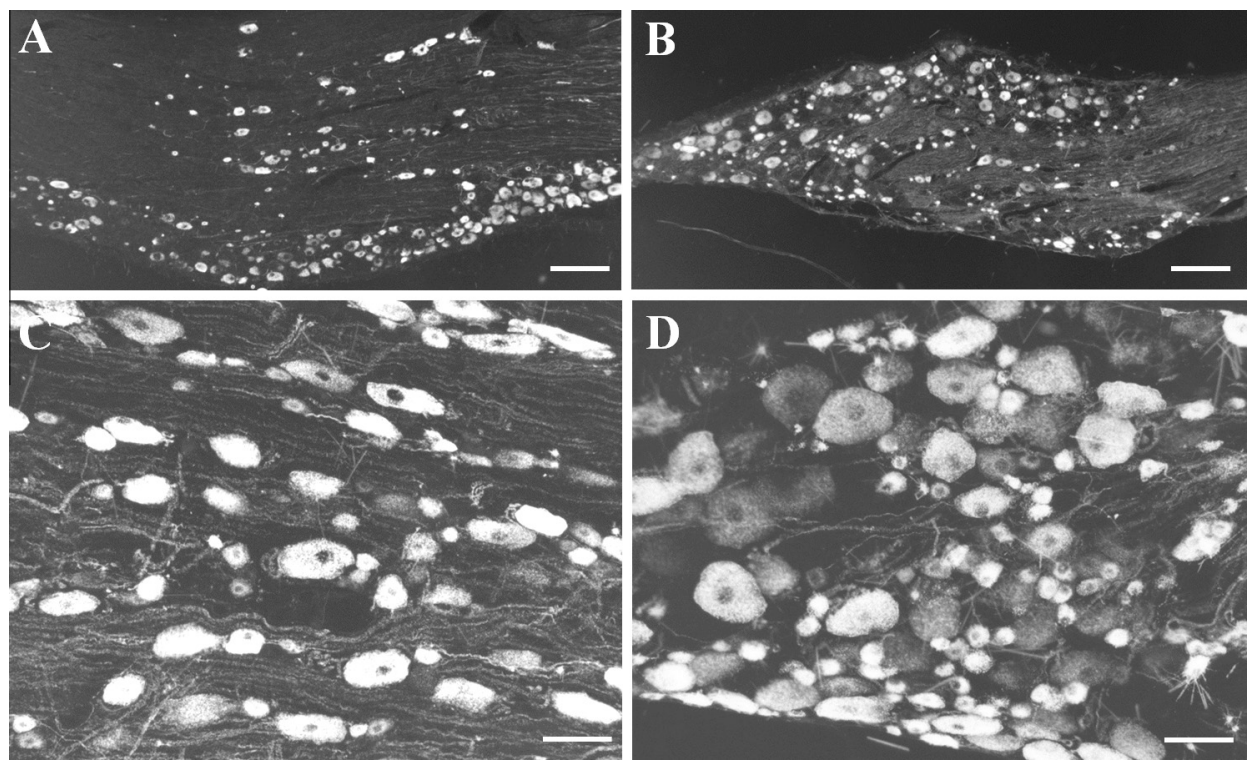

Fig. 1. Inverse microphotographs of labeled neurons in L5 dorsal root ganglia following the injection of CTB-HRP into intact (A, C) and capsaicintreated $(B, D)$ sciatic nerves of a rat. Note the marked increase in the number of labeled small neurons after capsaicin treatment. Scale bar indicates $200 \mu \mathrm{m}$ in $\mathrm{A}, \mathrm{B}$ and $50 \mu \mathrm{m}$ in $\mathrm{C}, \mathrm{D}$.

A

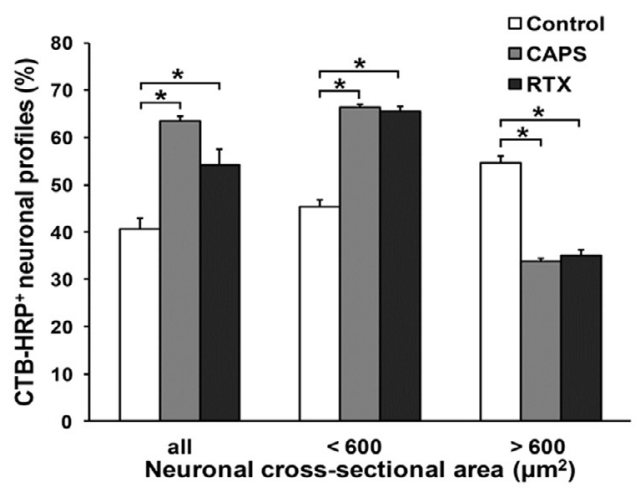

C

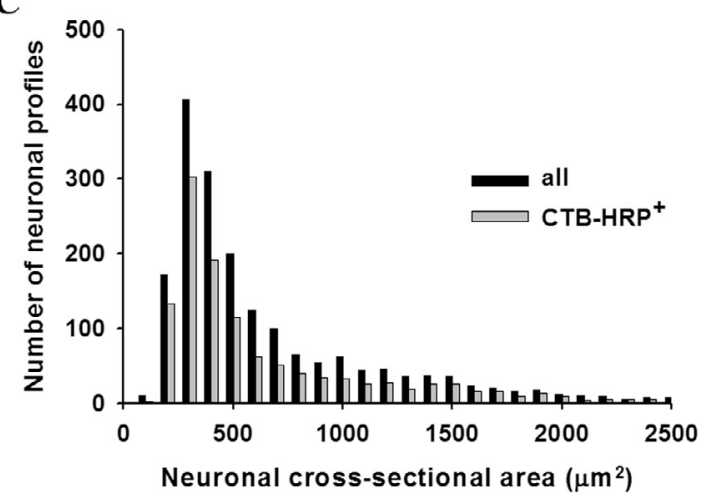

B

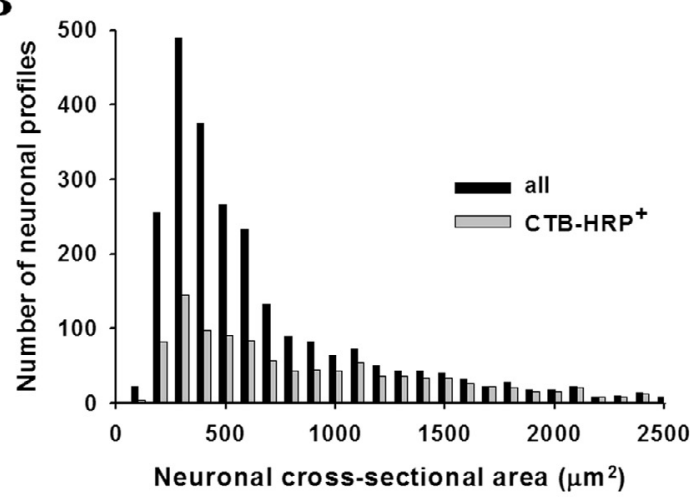

$\mathbf{D}$

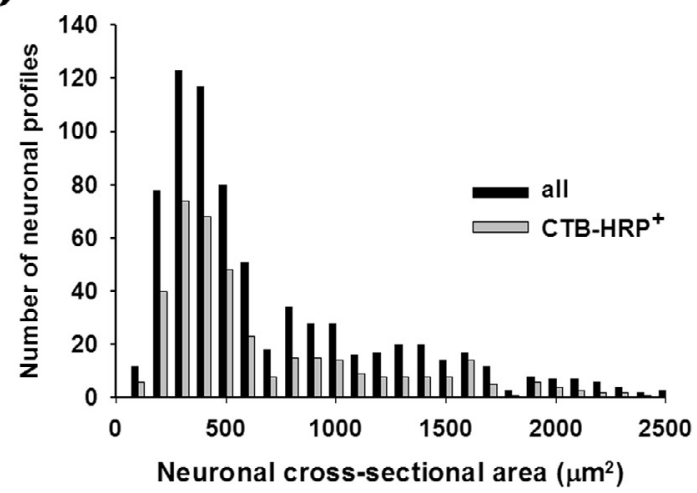

Fig. 2. (A) Proportions of different populations of CTB-HRP-labeled neurons in L5 dorsal root ganglia relating to the control, and capsaicin (CAPS)and resiniferatoxin (RTX)-treated sciatic nerves. The nerves were treated 2 weeks before the intraneural injection of the CTB-HRP tracer. *Significantly different from the control $(p<0.05, n=4)$. (B)-(D): Size-frequency distribution histograms of neurons in L5 dorsal root ganglia relating to control (B), and capsaicin- (C) or resiniferatoxin-treated (D) sciatic nerves. Note the marked increase in the number of CTB-HRP-labeled neuronal profiles after capsaicin and resiniferatoxin treatments. 

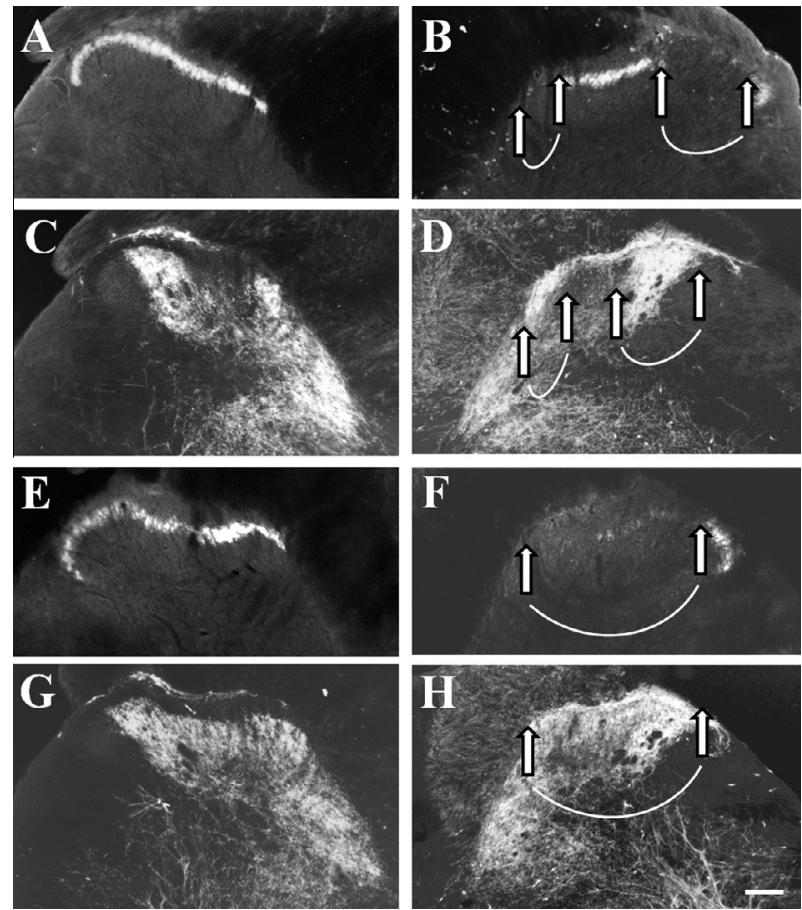

Fig. 3. Inverse photomicrographs illustrating the localization of thiamine monophosphatase enzyme activity (A, B, E, F) and the distribution of transganglionically transported CTB-HRP (C, D, G, H) within the rat spinal dorsal horn of the $3 \mathrm{rd}(\mathrm{A}-\mathrm{D})$ and 4 th $(\mathrm{E}-\mathrm{H})$ lumbar spinal cord segments 2 weeks after perineural capsaicin treatment. CTB-HRP was injected into intact sciatic nerves $(\mathrm{C}, \mathrm{G})$ or into sciatic nerves treated perineurally with capsaicin 2 weeks previously $(D, H)$. Injection of CTB-HRP into an intact nerve results in labeling of the deeper layers of the ipsilateral spinal dorsal horn (C, G), whereas after capsaicin treatment intense labeling is also present in the marginal zone and the substantia gelatinosa $(\mathrm{D}, \mathrm{H})$. Note the disappearance of thiamine monophosphatase activity in the substantia gelatinosa from the somatotopically appropriate regions of the sciatic nerve after capsaicin treatment (sites indicated by interconnected arrows). Also note that areas of CTB-HRP labeling of Rexed's laminae I-II are identical with sites of thiamine monophosphatase depletion as indicated by the interconnected arrows. The scale bar in $\mathrm{H}$ indicates $100 \mu \mathrm{m}$ and holds for all microphotographs.

\section{Effects of perineural treatment with capsaicin on the spinal distribution of unmyelinated sciatic primary afferents}

WGA and its conjugates are suitable tracer molecules with which to study the spinal distribution of unmyelinated primary afferent fibers. To evaluate possible structural alterations or changes in the projections of unmyelinated dorsal root fibers following perineural capsaicin treatment, therefore, the control and the capsaicin-treated sciatic nerves were injected with WGA-HRP. In control specimens, the peroxidase reaction product, indicating the presence of WGA-HRP, uniformly filled the substantia gelatinosa in a somatotopical manner throughout the L3-L6 segments of the spinal cord. Injection of this tracer into a capsaicin-treated nerve resulted in labeling of the substantia gelatinosa resembling that in the control in both topographical distribution and intensity (Fig. 5).
Effects of perineural treatment with capsaicin on the axonal transport of CTB-HRP by myelinated and unmyelinated dorsal root axons

Electron microscopy revealed that many myelinated axons in the control L5 dorsal roots displayed a distinct electron-dense reaction product localized either to the axolemma or to the axoplasm, or both. Very few labeled unmyelinated axons were seen in the control dorsal roots (Fig. 6A, D). The quantitative data disclosed that $50.6 \pm 6 \%$ of the myelinated, but only $3 \pm 1 \%$ of the unmyelinated dorsal root axons in the control dorsal roots transport CTB-HRP after injection into the sciatic nerve. Similarly, in the dorsal roots relating to the capsaicin-treated sciatic nerve, numerous myelinated axons contained electron-dense reaction product characteristic of HRP (Fig. 6E). However, in contrast with the control, many unmyelinated dorsal root axons were also labeled with CTB-HRP. The reaction product was localized to both the axolemma and the axoplasm (Fig. 6B, C, E). The quantitative data indicated that in the dorsal roots relating to the capsaicin-treated sciatic nerve, $57.2 \pm 6.5 \%$ of the myelinated and $47.8 \pm 6 \%$ of the unmyelinated axons transported the CTB-HRP conjugate. The difference between the proportions of labeled unmyelinated axons in the dorsal roots relating to the control and capsaicin-treated sciatic nerves was highly significant $(p<0.001, n=3)$.

\section{DISCUSSION}

The present study confirms and extends earlier findings by revealing a massive labeling by transganglionically transported CTB-HRP of the somatotopically relating regions of the substantia gelatinosa of the spinal dorsal horn of a peripheral nerve treated perineurally with vanilloid compounds, but not with their solvents. Previous studies suggested that the increased labeling of the substantia gelatinosa with CTB-HRP injected into a capsaicin-treated nerve might be accounted for by the sprouting of intact myelinated primary afferents relating to the treated nerve into that area, which is normally served only by unmyelinated primary afferent fibers (Mannion et al., 1996). The present study has confirmed this apparent "sprouting phenomenon" after either perineural capsaicin or resiniferatoxin treatment. However, perineural treatment with capsaicin or resiniferatoxin also resulted in an increase in the proportion of CTB-HRPlabeled small DRG neurons. This may be explained by an increase in intraneuronal GM1 ganglioside, the molecular target of CTB, which is specifically bound by this ganglioside. Most importantly, the transport of CTB-HRP by unmyelinated dorsal root axons has been demonstrated directly with electron microscopic histochemistry, showing that a significant proportion of unmyelinated dorsal root axons contain CTB-HRP following perineural capsaicin treatment, whereas such labeled fibers are only rarely seen in dorsal roots relating to the control nerve. Collectively, these observations indicate that, following perineural treatment with the TRPV1 agonists capsaicin or resiniferatoxin, $\mathrm{C}$-fiber primary afferent neurons undergo 

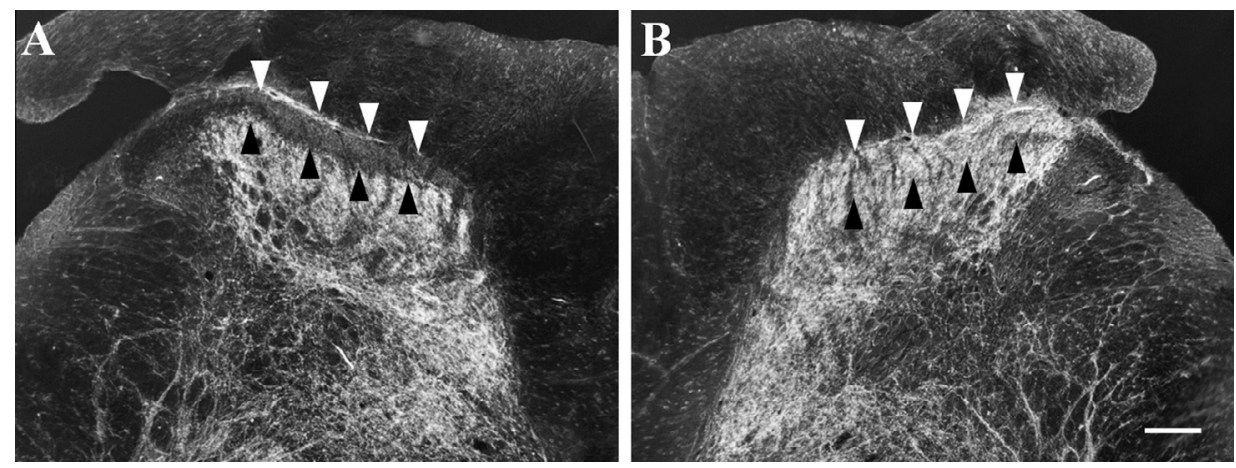

Fig. 4. Inverse photomicrographs illustrating the effect of perineural treatment with resiniferatoxin on the distribution of labeled primary afferents in the 4th lumbar spinal cord segment of the rat. CTB-HRP was injected into the intact sciatic nerve $(A)$ and into the contralateral sciatic nerve treated perineurally with resiniferatoxin 2 weeks previously (B). Note the massive labeling of the substantia gelatinosa (marked by arrowheads) following treatment with resiniferatoxin. The scale bar in B indicates $50 \mu \mathrm{m}$ and holds for both microphotographs.

a phenotypic switch and become capable of the uptake and transganglionic transport of CTB-HRP. This conclusion is supported by the finding that, similar to the control, injection of WGA-HRP into a capsaicin-treated sciatic nerve resulted in intense labeling of the somatotopically relating regions of the substantia gelatinosa, indicating the apparent anatomical integrity of unmyelinated primary afferents. This latter observation suggests that chemically injured unmyelinated primary afferents preserve their spatially constrained spinal terminal arborizations. Accordingly, the present results strongly suggest that the labeling of the substantia gelatinosa by CTB-HRP following perineural treatment with vanilloid compounds may be accounted for by a phenotypic switch of anatomically intact unmyelinated, C-fiber primary afferent neurons rather than a sprouting response of myelinated primary afferents. A similar conclusion was reached in earlier studies dealing with the consequences of peripheral nerve injuries, and in particular nerve transection, in terms of the transganglionic transport of CTB and its conjugates. These reports provided evidence in favor of the notion that, following transection of a peripheral nerve, CTB-HRP is taken up and, unlike in intact nerves, transported not only by myelinated, but also by unmyelinated primary afferents, and this may be responsible for the marked labeling of the substantia gelatinosa (Tong et al., 1999; Bao et al., 2002; Jancsó et al., 2002, 2004; Sántha and Jancsó, 2003; Shehab et al., 2003; Jancsó and Sántha, 2004). Further, these studies have found little support for the suggested massive sprouting of myelinated primary afferent fibers into the substantia gelatinosa after peripheral nerve transection (Bao et al., 2002; Shehab et al., 2003). This is also supported by the findings that, in the rat spinal dorsal horn, the plexus of vesicular glutamate transporter 1-immunoreactive boutons, which largely corresponds to the central terminals of large myelinated afferents, does not extend further dorsally after peripheral nerve transection (Hughes et al., 2004). Although these findings clearly demonstrated that extensive sprouting of myelinated primary afferents which bind CTB is unlikely to occur, electrophysiological findings have revealed the sprouting of some myelinated mechanoreceptor primary afferents after peripheral nerve injuries (Woolf et al., 1992; Koerber et al., 1994; Kohama et al., 2000; Woodbury et al., 2008). In view of the finding that a significant proportion of the small neurons is also labeled by CTB-HRP in control L5 ganglia, labeling of the substantia gelatinosa after perineural capsaicin may be effected by additional mechanisms affecting intraneuronal transport processes. It may be suggested that chemical perturbation of the sensory nerves by capsaicin interferes with mechanisms involved in regulation of the
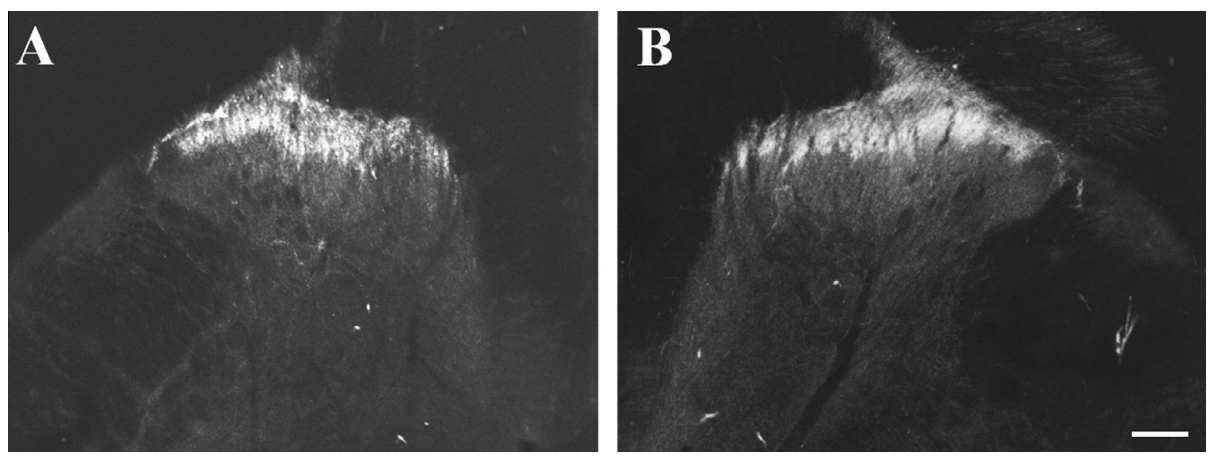

Fig. 5. Inverse photomicrographs illustrating the effect of perineural treatment with capsaicin on the distribution of labeled primary afferents in the substantia gelatinosa and marginal zone of 4 th lumbar spinal cord segment of the rat. WGA-HRP was injected into the intact sciatic nerve (A) and into the contralateral sciatic nerve treated perineurally with capsaicin 2 weeks previously (B). Note the similar distribution and intensity of the label ipsilaterally (B) and contralaterally (A) to the capsaicin-treated sciatic nerve. The scale bar in b indicates $50 \mu \mathrm{m}$ and holds for both microphotographs. 

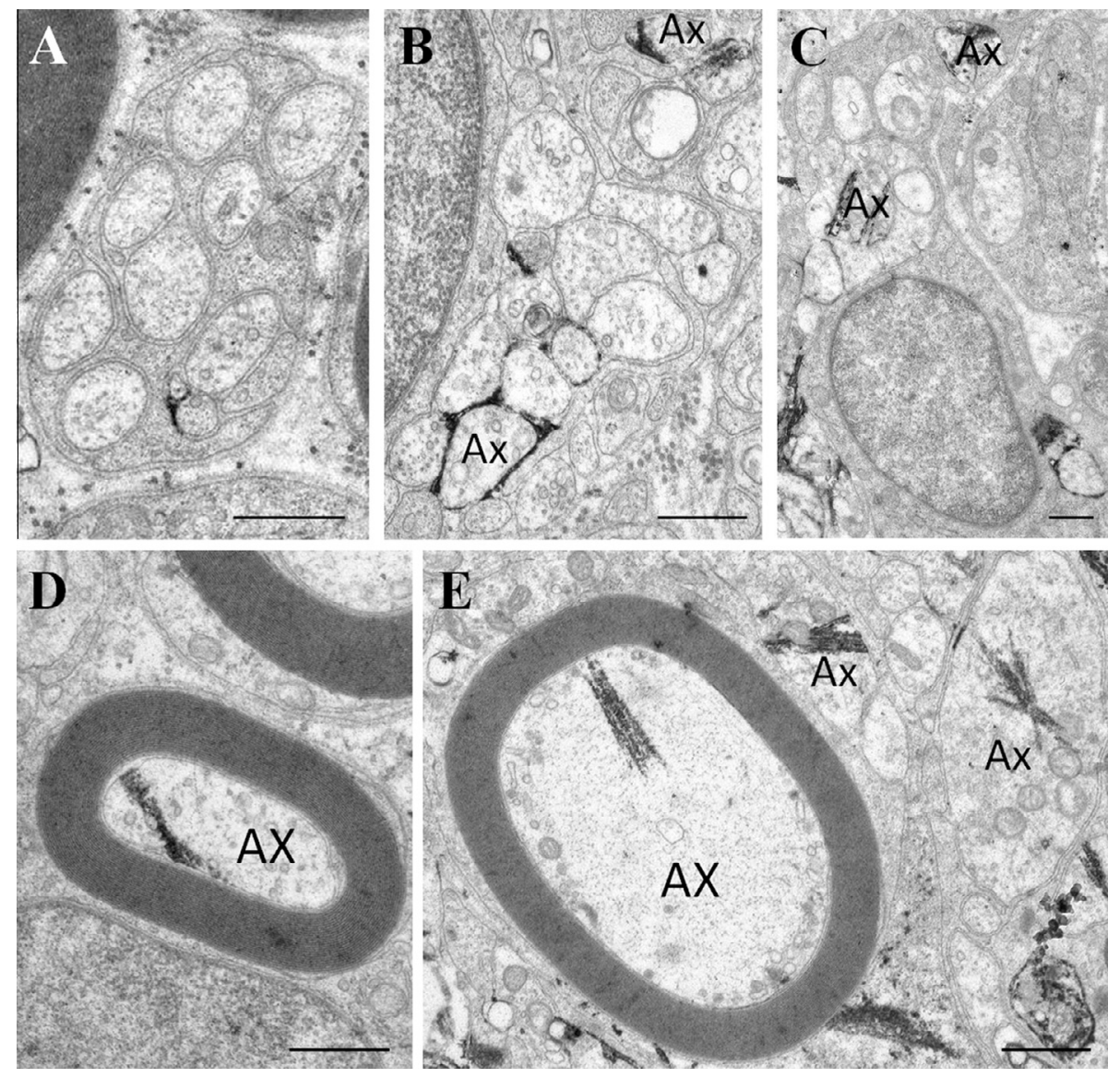

Fig. 6. Electron micrographs illustrating the localization of CTB-HRP in myelinated ( $A X)$ and unmyelinated $(A x)$ dorsal root axons relating to the intact $(A, D)$, capsaicin- $(B, C)$ and resiniferatoxin-treated $(E)$ sciatic nerves. The electron-dense product of the peroxidase reaction is either finely distributed and associated with the axolemma and/or localized as amorphous material within the axoplasm. The scale bars indicate $0.5 \mu \mathrm{m}$ in all figures.

cellular trafficking of specific molecules in primary sensory neurons. Hence, in neurons labeled by CTB-HRP in control ganglia, the perineural application of capsaicin may initiate changes resulting in spinopetal transport of tracer molecules toward the substantia gelatinosa. Further studies are needed to gain experimental support for this assumption.

The possible functional significance of the increased uptake and transport of CTB-HRP by unmyelinated primary afferent neurons following perineural treatment with capsaicin is difficult to envisage at present. Although CTB is largely regarded solely as a neuronal tracer, it is a highly specific biochemical marker for the GM1 ganglioside. It has therefore been suggested that the GM1 ganglioside may play a significant role in the function of nociceptive primary sensory neurons (Sántha and Jancsó, 2003; Jancsó and Sántha, 2004). Indeed, it has been demonstrated that pharmacological manipulation of the neuronal GM1 ganglioside level of cultured adult dorsal root ganglion neurons interferes significantly with the nociceptor function. Inhibition of glucosylceramide synthase resulted in a reduced neuronal GM1 level and significant reductions in neuronal capsaicin sensitivity and TRPV1 expression (Sántha et al., 2010). Moreover, the acute sensitization of cultured nociceptive sensory ganglion cells by the nerve growth factor was inhibited after the depletion of neuronal gangliosides. Local regulatory (efferent) nociceptor functions were also affected, as revealed by a marked reduction of the capsaicin-, but not the high potassium-induced release of calcitonin gene-related peptide (CGRP) following the inhibition of glucosylceramide synthase. There are at least two possible mechanisms which may underlie these functional changes. First, the gangliosides, and in particular GM1, are involved in mediation of the effects of trophic factors such as the nerve growth factor, which in turn is essential in the regulation of the expression of the TRPV1 receptor in DRG neurons (Wood et al., 1988; Michael and Priestley, 1999). A close association with and activation of the TrkA receptor by GM1 has also been reported and suggested to contribute to the mechanism of GM1 ganglioside potentiation of nerve growth factor (NGF) action (Leon et al., 1984; Mutoh et al., 1995; Duchemin et al., 2008; Ledeen and Wu, 2015). Second, gangliosides are important constituents of membrane lipid rafts whose association with membrane receptors, including TRPV1, may play a significant role in the activation of that nociceptor channel (Sántha and Jancsó, 2003; Liu et al., 2006; Sántha et al., 2010; Szoke et al., 2010). It has additionally been demonstrated that overexpression of the GM1 ganglioside suppressed nerve growth factor signals in PC12 cells (Nishio et al., 2004), an observation 
that may explain the apparently contrasting cellular and functional changes brought about by perineural capsaicin treatment. Hence, a marked increase in neuronal GM1 ganglioside expression may impede nerve growth factor signaling and consequently the expression of TRPV1, which is critically dependent on nerve growth factor. A decrease in the expression level of the TRPV1 receptor may explain the impairment of nociceptive functions and the development of nociceptor analgesia observed after perineural capsaicin (Jancsó et al., 1980; Fitzgerald and Woolf, 1982) or resiniferatoxin (Kissin et al., 2002; Kissin, 2008) treatments. Indeed, recent findings furnished evidence of a decrease in TRPV1 mRNA and a massive permanent reduction in TRPV1 protein of DRG neurons following perineural treatment with capsaicin (Szigeti et al., 2012).

The results of the present study have important implications as regards the possible anatomical and neurochemical hallmarks of neuropathic pain, an impaired sensory function and nociceptor analgesia. It appears that the changes demonstrated after peripheral nerve lesions or experimental perturbations in models of chronic/neuropathic pain and analgesia, respectively, are apparently identical or similar, at least at the phenomenological level. These alterations include decreases in the levels of sensory neuropeptides, e.g. substance $P(S P)$ and CGRP, reduced expression of the TRPV1 receptor, depletion of TMP and reduction of Griffonia simplicifolia isolectin B4 (IB4) binding in the DRGs and the substantia gelatinosa (Jancsó, 1992; Fazen and Ringkamp, 2008; Sandkuhler, 2009; Kuner, 2010). These findings clearly suggest that many of the anatomical and neurochemical changes believed to be specific for injuries of primary afferent neurons also occur after perineural treatment with capsaicin or resiniferatoxin, which induce selective regional chemical and thermal analgesia (Fitzgerald and Woolf, 1982; Gamse et al., 1982; Chung et al., 1985; Jancsó et al., 1987b). This indicates that many of the cellular processes involved in the development of pain and analgesia may surprisingly, impact upon common cellular mechanisms. Hence, these anatomical and neurochemical alterations cannot be regarded as changes specific for peripheral nerve injuries. It must be noted that, although perineural treatment with vanilloid compounds may also cause certain changes which may be interpreted in terms of (chemical) nerve damage, these develop only after much longer post-treatment periods of several weeks (Jancsó and Lawson, 1990; Pini and Lynn, 1991) than the analgesia which develops within hours (Jancsó et al., 1980; Fitzgerald and Woolf, 1982). In different models of neuropathic pain and vanilloid-induced nociceptor analgesia, respectively, axonal transport processes may be compromised (Gamse et al., 1982; Thakor et al., 2009; Dilley et al., 2013; Maiya and Messing, 2014), resulting in a lack or decreased availability of trophic factors, such as the nerve growth factor. This may result, among others in the down-regulation of sensory neuropeptides and, importantly, nociceptor ion channels, such as the TRPV1 channel (Michael and Priestley, 1999; Szigeti et al., 2012).

\section{CONCLUSION}

The present findings have provided evidence of a substantial, previously unrecognized phenotypic change of C-fiber primary sensory neurons following perineural treatment with vanilloid compounds that produce selective chemical and thermal analgesia. The results demonstrate that the increased labeling by CTB-HRP of the substantia gelatinosa after perineural capsaicin or resiniferatoxin treatment may be attributed to an increased uptake and transganglionic transport of CTBHRP by $\mathrm{C}$-fiber nociceptive primary afferent neurons rather than a sprouting response of myelinated primary afferents. It is further suggested that modulation of the expression of GM1 ganglioside in C-fiber nociceptive neurons may be of significance in terms of the regulation of the expression of the archetypal nociceptive ion channel, the TRPV1 receptor. It is assumed therefore that pharmacological manipulation of the neuronal GM1 ganglioside level may serve as a novel approach to regulation of the expression of nociceptive TRP channels and to pain relief.

Acknowledgments-This work was supported by research Grant K-101873 of the Hungarian Scientific Research Fund (OTKA). Péter Sántha was supported by a János Bolyai Research Fellowship from the Hungarian Academy of Sciences.

\section{REFERENCES}

Ainsworth A, Hall P, Wall PD, Allt G, MacKenzie ML, Gibson S, Polak JM (1981) Effects of capsaicin applied locally to adult peripheral nerve. II. Anatomy and enzyme and peptide chemistry of peripheral nerve and spinal cord. Pain 11:379-388.

Bao L, Wang HF, Cai HJ, Tong YJ, Jin SX, Lu Y, Grant G, Hökfelt T, Zhang $X$ (2002) Peripheral axotomy induces only very limited sprouting of coarse myelinated afferents into inner lamina II of rat spinal cord. Eur J Neurosci 16(2):175-185.

Brown AG (1981) Organization of the spinal cord. The anatomy and physiology of identified neurones. Berlin: Springer-Verlag.

Caterina MJ, Schumacher MA, Tominaga M, Rosen TA, Levine JD, Julius D (1997) The capsaicin receptor: a heat-activated ion channel in the pain pathway. Nature 389(6653):816-824.

Chung JM, Lee KH, Hori Y, Willis WD (1985) Effects of capsaicin applied to a peripheral nerve on the responses of primate spinothalamic tract cells. Brain Res 329:27-38.

Crain SM, Shen KF (1998) Modulation of opioid analgesia, tolerance and dependence by Gs-coupled, GM1 ganglioside-regulated opioid receptor functions. Trends Pharmacol Sci 19:358-365.

Cuatrecasas P (1973) Gangliosides and membrane receptors for cholera toxin. Biochemistry 12:3558-3566.

Dilley A, Richards N, Pulman KG, Bove GM (2013) Disruption of fast axonal transport in the rat induces behavioral changes consistent with neuropathic pain. J Pain 14:1437-1449.

Duchemin AM, Ren Q, Neff NH, Hadjiconstantinou M (2008) GM1induced activation of phosphatidylinositol 3-kinase: involvement of Trk receptors. J Neurochem 104:1466-1477.

Eidels L, Proia RL, Hart DA (1983) Membrane receptors for bacterial toxins. Microbiol Rev 47:596-620.

Fazen LE, Ringkamp M (2008) The pathophysiology of neuropathic pain: a review of current research and hypotheses. Neurosurg $Q$ 17:245-262.

Fitzgerald M, Woolf CJ (1982) The time course and specificity of the changes in the behavioural and dorsal horn cell responses to 
noxious stimuli following peripheral nerve capsaicin treatment in the rat. Neuroscience 7:2051-2056.

Gamse R, Petsche U, Lembeck F, Jancsó G (1982) Capsaicin applied to peripheral nerve inhibits axoplasmic transport of substance $\mathrm{P}$ and somatostatin. Brain Res 239:447-462.

Gibson SJ, McGregor G, Bloom SR, Polak JM, Wall PD (1982) Local application of capsaicin to one sciatic nerve of the adult rat induces a marked depletion in the peptide content of the lumbar dorsal horn. Neuroscience 7(12):3153-3162.

Gu Y, Chen Y, Ye L (1992) Electron microscopical demonstration of horseradish peroxidase by use of tetramethylbenzidine as chromogen and sodium tungstate as stabilizer (TMB-ST method): a tracing method with high sensitivity and well preserved ultrastructural tissue. J Neurosci Methods 42:1-10.

Holzer P (1991) Capsaicin: cellular targets, mechanisms of action, and selectivity for thin sensory neurons. Pharmacol Rev 43:143-201.

Hughes DI, Polgár E, Shehab SA, Todd AJ (2004) Peripheral axotomy induces depletion of the vesicular glutamate transporter VGLUT1 in central terminals of myelinated afferent fibres in the rat spinal cord. Brain Res 1017:69-76.

Hughes DI, Scott DT, Todd AJ, Riddell JS (2003) Lack of evidence for sprouting of Abeta afferents into the superficial laminas of the spinal cord dorsal horn after nerve section. J Neurosci 23:9491-9499.

Inomata K, Ogawa K (1979) Ultracytochemical localization of thiamine monophosphatase activity in the substantia gelatinosa of the rat spinal cord. Acta Histochem Cytochem 12:337.

Jancsó G (1992) Pathobiological reactions of C-fibre primary sensory neurones to peripheral nerve injury. Exp Physiol 77:405-431.

Jancsó G, Ambrus A (1994) Capsaicin sensitivity of primary sensory neurones and its regulation. In: Besson J, Guilbaud G, Ollat H, editors. Peripheral neurons in nociception: physiopharmacological aspects. Paris: John Libbey Eurotext. p. 71-87.

Jancsó G, Lawson SN (1990) Transganglionic degeneration of capsaicin-sensitive C-fiber primary afferent terminals. Neuroscience 39:501-511.

Jancsó G, Lynn B (1987) Possible use of capsaicin in pain therapy. Clin J Pain 3:123-126.

Jancsó G, Sántha P (2004) Transganglionic transport of choleragenoid by injured $\mathrm{C}$ fibers to the substantia gelatinosa: relevance to neuropathic pain and hyperalgesia. In: Brune K, Handwerker $\mathrm{HO}$, editors. Hyperalgesia: molecular mechanisms and clinical implications. Seattle: IASP Press. p. 143-156.

Jancsó G, Sántha P (2015) The foundation of sensory pharmacology: Nicholas (Miklós) Jancsó and the Szeged contribution. Temperature 2:152-157.

Jancsó G, Such G (1983) Effects of capsaicin applied perineurally to the vagus nerve on cardiovascular and respiratory functions in the cat. J Physiol 341:359-370.

Jancsó G, Kiraly E, Jancsó-Gábor A (1980) Direct evidence for an axonal site of action of capsaicin. Naunyn-Schmiedebergs Arch Pharmacol 313:91-94.

Jancsó G, Ferencsik M, Such G, Kiraly E, Nagy A (1985) Morphological effects of capsaicin and its analogues in newborn and adult mammals. In: Haikanson R, Sundler F, editors. Tachykinin Antagonists. Amsterdam: Elsevier. p. 35-44.

Jancsó G, Kiraly E, Joó F, Such G, Nagy A (1987a) Neurotoxic effect of capsaicin in mammals. Acta Physiol Hung 69:295-313.

Jancsó G, Such G, Rödel C (1987b) A new approach to selective regional analgesia. In: Sicuteri $F$, Vecchiet $L$, Fanciullacci $M$, editors. Trends in cluster headache. Amsterdam: Elsevier. p. $59-68$.

Jancsó G, Sántha P, Gecse K (2002) Peripheral nerve lesioninduced uptake and transport of choleragenoid by capsaicinsensitive c-fibre spinal ganglion neurons. Acta Biol Hung 53(1-2):77-84.

Jancsó G, Sántha P, Szigeti C, Dux M (2004) Selective C-fiber deafferentation of the spinal dorsal horn prevents lesion-induced transganglionic transport of choleragenoid to the substantia gelatinosa in the rat. Neurosci Lett 361:204-207.
Jancsó G, Dux M, Oszlács O, Sántha P (2008) Activation of the transient receptor potential vanilloid-1 (TRPV1) channel opens the gate for pain relief. $\mathrm{Br} \mathrm{J}$ Pharmacol 155:1139-1141.

Jancsó G, Oszlács O, Sántha P (2011) The capsaicin paradox: pain relief by an analgesic agent. Antiinflamm Antiallergy Agents Med Chem 10:52-65.

Kissin I (2008) Vanilloid-induced conduction analgesia: selective, dose-dependent, long-lasting, with a low level of potential neurotoxicity. Anesth Analg 107:271-281.

Kissin I, Bright C, Bradley Jr EL (2002) Selective and long-lasting neural blockade with resiniferatoxin prevents inflammatory pain hypersensitivity. Anesth Analg 94:1253-1258.

Knotkova H, Pappagallo M, Szallasi A (2008) Capsaicin (TRPV1 Agonist) therapy for pain relief: farewell or revival? Clin J Pain 24:142-154.

Koerber HR, Mirnics K, Brown PB, Mendell LM (1994) Central sprouting and functional plasticity of regenerated primary afferents. J Neurosci 14:3655-3671.

Kohama I, Ishikawa K, Kocsis JD (2000) Synaptic reorganization in the substantia gelatinosa after peripheral nerve neuroma formation: aberrant innervation of lamina II neurons by Abeta afferents. J Neurosci 20:1538-1549.

Kuner R (2010) Central mechanisms of pathological pain. Nat Med 16:1258-1266.

LaMotte CC, Kapadia SE, Shapiro CM (1991) Central projections of the sciatic, saphenous, median, and ulnar nerves of the rat demonstrated by transganglionic transport of choleragenoid-HRP (B-HRP) and wheat germ agglutinin-HRP (WGA-HRP). J Comp Neurol 311:546-562.

Ledeen RW, Wu G (2015) The multi-tasked life of GM1 ganglioside, a true factotum of nature. Trends Biochem Sci 40:407-418.

Leon A, Benvegnu D, Dal Toso R, Presti D, Facci L, Giorgi O, Toffano G (1984) Dorsal root ganglia and nerve growth factor: a model for understanding the mechanism of GM1 effects on neuronal repair. J Neurosci Res 12:277-287.

Liu M, Huang W, Wu D, Priestley JV (2006) TRPV1, but not P2X, requires cholesterol for its function and membrane expression in rat nociceptors. Eur J Neurosci 24:1-6.

Lynn B, Pini A (1984) Axonal recovery and development of a longlasting peripheral block in C-fibre polymodal nociceptors after local nerve treatment with capsaicin in the rat. J Physiol 350:34.

Maggi CA, Lippe IT, Giuliani S, Abelli L, Somma V, Geppetti P, Jancsó G, Santicioli P, Meli A (1989) Topical versus systemic capsaicin desensitization: specific and unspecific effects as indicated by modification or reflex micturition in rats. Neuroscience 31(3):745-756.

Maiya RP, Messing RO (2014) Peripheral systems: neuropathy. Handb Clin Neurol 125:513-525.

Mannion RJ, Doubell TP, Coggeshall RE, Woolf CJ (1996) Collateral sprouting of uninjured primary afferent A-fibers into the superficial dorsal horn of the adult rat spinal cord after topical capsaicin treatment to the sciatic nerve. J Neurosci 16:5189-5195.

Mesulam MM (1978) Tetramethyl benzidine for horseradish peroxidase neurohistochemistry: a non-carcinogenic blue reaction product with superior sensitivity for visualizing neural afferents and efferents. J Histochem Cytochem 26:106-117.

Michael GJ, Priestley JV (1999) Differential expression of the mRNA for the vanilloid receptor subtype 1 in cells of the adult rat dorsal root and nodose ganglia and its downregulation by axotomy. J Neurosci 19:1844-1854.

Mutoh T, Tokuda A, Miyadai T, Hamaguchi M, Fujiki N (1995) Ganglioside GM1 binds to the Trk protein and regulates receptor function. Proc Natl Acad Sci U S A 92:5087-5091.

Nagy I, Sántha P, Jancsó G, Urbán L (2004) The role of the vanilloid (capsaicin) receptor (TRPV1) in physiology and pathology. Eur $\mathrm{J}$ Pharmacol 500:351-369.

Nishio M, Fukumoto S, Furukawa K, Ichimura A, Miyazaki H, Kusunoki S, Urano T, Furukawa K (2004) Overexpressed GM1 suppresses nerve growth factor (NGF) signals by modulating the intracellular localization of NGF receptors and membrane fluidity in PC12 cells. J Biol Chem 279:33368-33378. 
Petsche U, Fleischer E, Lembeck F, Handwerker HO (1983) The effect of capsaicin application to a peripheral nerve on impulse conduction in functionally identified afferent nerve fibres. Brain Res 265:233-240.

Pini A, Lynn B (1991) C-fibre function during the 6 weeks following brief application of capsaicin to a cutaneous nerve in the rat. Eur $\mathrm{J}$ Neurosci 3:274-284.

Ramon y Cajal S (1995) Histology of the nervous system of man and vertebrates. New York: Oxford University Press. vol. in two volumes.

Réthelyi M, Szentágothai J (1973) Distribution and connections of afferent fibers in the spinal cord. In: Iggo A, editor. Handbook of sensory physiology, vol. 2. Berlin: Springer-Verlag. p. 237-252.

Rivero-Melian C, Grant G (1991) Choleragenoid horseradish peroxidase used for studying projections of some hindlimb cutaneous nerves and plantar foot afferents in the dorsal horn and Clarke's column in the rat. Exp Brain Res 84:125-132.

Robertson B, Grant G (1985) A comparison between wheat germ agglutinin-and choleragenoid-horseradish peroxidase as anterogradely transported markers in central branches of primary sensory neurones in the rat with some observations in the cat. Neuroscience 14:895-905.

Sandkuhler J (2009) Models and mechanisms of hyperalgesia and allodynia. Physiol Rev 89:707-758.

Sántha P, Jancsó G (2003) Transganglionic transport of choleragenoid by capsaicin-sensitive C-fibre afferents to the substantia gelatinosa of the spinal dorsal horn after peripheral nerve section. Neuroscience 116(3):621-627.

Sántha P, Oszlács O, Dux M, Dobos I, Jancsó G (2010) Inhibition of glucosylceramide synthase reversibly decreases the capsaicininduced activation and TRPV1 expression of cultured dorsal root ganglion neurons. Pain 150:103-112.

Shehab SA, Spike RC, Todd AJ (2003) Evidence against cholera toxin B subunit as a reliable tracer for sprouting of primary afferents following peripheral nerve injury. Brain Res 964 (2):218-227.

Soares S, von Boxberg Y, Lombard M, Ravaille-Veron M, Fischer I, Eyre J, Nothias F (2002) Phosphorylated MAP1B is induced in central sprouting of primary afferents in response to peripheral injury but not in response to rhizotomy. Eur $J$ Neurosci 16:593-606.

South EH, Ritter RC (1988) Capsaicin application to central or peripheral vagal fibers attenuates CCK satiety. Peptides 9:601-612.
Szentágothai J (1964) Neuronal and synaptic arrangement in the substantia gelatinosa rolandi. J Comp Neurol 122:219-239.

Szigeti C, Sántha P, Körtvély E, Nyári T, Horváth VJ, Deák É, Dux M, Gulya K, Jancsó G (2012) Disparate changes in the expression of transient receptor potential vanilloid type 1 receptor mRNA and protein in dorsal root ganglion neurons following local capsaicin treatment of the sciatic nerve in the rat. Neuroscience 10:320-330.

Szoke E, Börzsei R, Tóth DM, Lengl O, Helyes Z, Sándor Z, Szolcsányi J (2010) Effect of lipid raft disruption on TRPV1 receptor activation of trigeminal sensory neurons and transfected cell line. Eur J Pharmacol 628:67-74.

Thakor DK, Lin A, Matsuka Y, Meyer EM, Ruangsri S, Nishimura I, Spigelman I (2009) Increased peripheral nerve excitability and local NaV1.8 mRNA up-regulation in painful neuropathy. Mol Pain 5:14.

Tong YG, Wang HF, Ju G, Grant G, Hökfelt T, Zhang X (1999) Increased uptake and transport of cholera toxin B-subunit in dorsal root ganglion neurons after peripheral axotomy: possible implications for sensory sprouting. J Comp Neurol 404:143-158.

Welk E, Petsche U, Fleischer E, Handwerker HO (1983) Altered excitability of afferent $\mathrm{C}$-fibers of the rat distal to a nerve site exposed to capsaicin. Neurosci Lett 38:245-250.

Willis WD, Coggeshall RE (1991) Sensory mechanisms of the spinal cord. New York: Plenum Press.

Winter J, Forbes CA, Sternberg J, Lindsay RM (1988) Nerve growth factor (NGF) regulates adult rat cultured dorsal root ganglion neuron responses to the excitotoxin capsaicin. Neuron 1:973-981.

Wood JN, Winter J, James IF, Rang HP, Yeats J, Bevan S (1988) Capsaicin-induced ion fluxes in dorsal root ganglion cells in culture. J Neurosci 8:3208-3220.

Woodbury CJ, Kullmann FA, Mcllwrath SL, Koerber HR (2008) Identity of myelinated cutaneous sensory neurons projecting to nocireceptive laminae following nerve injury in adult mice. J Comp Neurol 508:500-509.

Woolf CJ, Shortland P, Coggeshall RE (1992) Peripheral nerve injury triggers central sprouting of myelinated afferents. Nature 355:75-78.

Yip HK, Rich KM, Lampe PA, Johnson Jr EM (1984) The effects of nerve growth factor and its antiserum on the postnatal development and survival after injury of sensory neurons in rat dorsal root ganglia. J Neurosci 4:2986-2992. 